\title{
A Confirmatory Method Based on HPLC-MS/MS for the Detection and Quantification of Residue of Tetracyclines in Nonmedicated Feed
}

\author{
Rosa E. Gavilán, Carolina Nebot, Maria Veiga-Gómez, Paula Roca-Saavedra, \\ Beatriz Vazquez Belda, Carlos M. Franco, and Alberto Cepeda
}

Department of Analytical Chemistry, Nutrition and Bromatology, Faculty of Veterinary Medicine, University of Santiago de Compostela, 27002 Lugo, Spain

Correspondence should be addressed to Carolina Nebot; carolina.nebot@usc.es

Received 25 February 2016; Accepted 5 July 2016

Academic Editor: Karoly Heberger

Copyright @ 2016 Rosa E. Gavilán et al. This is an open access article distributed under the Creative Commons Attribution License, which permits unrestricted use, distribution, and reproduction in any medium, provided the original work is properly cited.

\begin{abstract}
The Commission Regulation 574/2011/EC set up maximum levels of coccidiostats and histomonostats in nonmedicated feed as a consequence of carry-over during manufacturing. Carry-over takes place from medicated to nonmedicated feed during feed production. Similar contamination could also occur for other pharmaceuticals such as tetracyclines, a group of antibiotics commonly employed in food production animal. The objective of this work is to present a simple and fast method for the simultaneous detection of four tetracyclines (chlortetracycline, doxycycline, oxytetracycline, and tetracycline) in nontarget feed at a $\mu \mathrm{g} / \mathrm{kg}$ level. Validation of the method was performed according to the guideline included in the Commission Decision 2002/657/EC for official method. The validated method was successfully applied to 50 feed samples collected from different milk farms and 25 samples obtained from feed manufacturers. While oxytetracycline was the tetracycline most frequently detected, chlortetracycline was the analyte measured at the highest concentration $15.14 \mathrm{mg} / \mathrm{Kg}$. From 75 nonmedicated feed analysed $15 \%$ resulted to be positive for the presence of one tetracycline.
\end{abstract}

\section{Introduction}

The demand for food of animal origin increases each year. To satisfy this demand, livestock production within the European Union (EU) in 2011 was approximately 10 million heads of goats, 80 million heads of sheep, 80 million heads of cattle, and 150 million heads of pigs [1]. To produce safe and nutritional food products, the animals need to be in good health. Like humans, animals also get sick and need medicines. Even though veterinary medicines contribute to improving and maintaining animal health, administration of these medicines by the farmer is carried out under licence via a veterinarian. The amount of drugs employed in food production estimated by Kools et al. was $6051 \mathrm{t}$, with antibiotics the most frequently used class of drug (5393 t) [2]. The groups of tetracyclines and $\beta$-lactams were used in high amounts and antiparasitic agents (194t) were the second most frequently used class of drug.
Intensively produced animals are often fed with concentrated feed, a mixture of various materials (oats, wheat, barley, rye, cottonseed, and crambe) and additives. Antibiotic and antiparasitic agents are the classes of drugs most commonly administrated. Antibiotic sulphonamides, tetracyclines, and $\beta$-lactam are the most frequently used antibiotics, and coccidiostats and ivermectin are the most frequently used antiparasitic agents. Coccidiostats and histomonostats are a group of antiparasitic agents that have been shown to be persistent during the manufacture of feed and carryover of this type of drugs has been demonstrated. The EU introduced maximum levels for these substances in nontarget feed in 2009 [3]; this regulation was later modified for some coccidiostats by the Regulation EC/574/2011 [4].

Cross contamination between medicated and nonmedicated feed could occur with any type of drug added to the feed, not only to coccidiostats, particularly when the cleaning process between batches is inefficient. Recently, 
a study conducted by Stolker et al. in the Netherlands confirmed that nonmedicated feed batches were contaminated with antibiotic residues such as tetracyclines, penicillins, and sulfonamides [5]. From 140 samples analysed, 87\% tested positive for antibiotics with a concentration of $0.1-154 \mathrm{mg} / \mathrm{kg}$. The fact that antibiotics could be present as contaminants in feed without the farmers' knowledge implies that withdrawal times will not be considered and antibiotic residues could remain in animal products (meat, eggs, milk, honey, seafood, and fish), in addition to the development of antibiotic resistant bacteria [6].

Due to the problems related to food safety, the authorities involved regularly monitor the presence of veterinary drugs in food of animal origin (eggs, milk, muscle, and liver). Controls on the water and food consumed by the animals have also been implemented, but the analysis conducted only evaluated the presence of substances such as pesticides [7, 8], nitrofurans [9], and mycotoxins [10, 11]. Methods based on HPLC-MS/MS detection are considered confirmatory methods because these types of methods provide full or complementary information, enabling substances to be unequivocally identified and if necessary quantified at the level of interest, according to the European Commission Decision 2002/657/EC [12]. Therefore, it is recommended to use confirmatory methods to detect the presence of antibiotics in nontarget feed. Few methods can be found in the scientific literature with these characteristics. Van Poucke et al. reported a method for the analysis of zinc bacitracin, spiramycin, tylosin, and virginiamycin with quantification limits below $500 \mu \mathrm{g} / \mathrm{kg}$ [13]. Boscher et al. published a multiclass method for the analysis of 33 analytes for 14 groups of antibiotics (including tetracyclines, quinolones, penicillins, ionophore coccidiostats, macrolides, and sulphonamides) with quantification limits of $3.8-65.0 \mu \mathrm{g} / \mathrm{kg}$ [14]. More recently, a method reported by Stolker et al. was able to measure tetracyclines, macrolides, sulphonamides, and penicillins [5].

Of the eight forms of commercially available tetracyclines, four are frequently used in food animal production (chlortetracycline, doxycycline, oxytetracycline, and tetracycline). Maximum residue limits (MRL) for these four tetracyclines and their three epimers have been introduced for various foods of animal origin, including eggs, muscle, and milk [15]. As maximum levels (ML) for these substances in feed samples have not been established, their complete absence is expected. Concentrations of tetracyclines in medicated feed are variable and depend on the target animal, with dosage rates between 25 and $700 \mathrm{mg} / \mathrm{kg}$. Therefore, carry-over should be expected in the batch of feed manufactured after a medicated feed. Stolker et al. reported that $100 \%$ of samples from the first batch of feed produced after the manufacture of medicated feed were contaminated with tetracyclines, with concentrations of $0.5-154 \mathrm{mg} / \mathrm{Kg}$ [5]. Based on these results, carry-over contamination is also expected for the other two commonly used tetracyclines (chlortetracycline and tetracycline) as they have similar chemical properties [16, 17].

Based on the common use of tetracyclines in food animal production and the absence of confirmatory methods for the presence of the four tetracyclines in animal feed, the aim of this work is to present an HPLC-MS/MS method for the analysis of tetracyclines in nonmedicated feed samples at levels of $\mu \mathrm{g} / \mathrm{kg}$.

\section{Experimental}

2.1. Chemicals, Reagents, and Stock Solutions. Disodium hydrogen phosphate dehydrate, anhydrous citric acid, ethylenediaminetetraacetic acid disodium salt (EDTA), trichloroacetic acid (TCA), and formic acid (purity $>99 \%$ by analysis) were purchased from Sigma-Aldrich (MO, USA). Tetracycline, chlortetracycline, doxycycline, and oxytetracycline (purity > 98\%) and demeclocycline, used as the internal standard (IS), were supplied by Sigma-Aldrich (MO, USA).

Organic solvents, methanol and ethyl acetate, HPLC or analytical grade, were purchased from Scharlau Chemie (Barcelona, Spain) and demineralised water (resistivity 18 $\mathrm{MU} \mathrm{cm}$ ) was prepared in-house with a Milli-Q water system (Millipore, Bedford, MA, USA).

Mobile phase A consisted of Milli-Q water acidified to $0.04 \%$ with formic acid and mobile phase B consisted of methanol, acidified to $0.1 \%$ with formic acid. To prepare the individual stock solution of tetracycline, $20 \mathrm{mg}$ of tetracycline was dissolved in $20 \mathrm{~mL}$ of methanol and stored at $-20^{\circ} \mathrm{C}$ for up to six months. The intermediate solution, a mixture of tetracyclines, was prepared by diluting the stock solution of each tetracycline to a final concentration of $50 \mu \mathrm{g} / \mathrm{mL}$ and stored at $-20^{\circ} \mathrm{C}$ for up to one month. A standard working solution of tetracycline was prepared freshly each day by diluting the intermediate stock solution to a final concentration of $1 \mu \mathrm{g} / \mathrm{mL}$. For the internal standard stock solution, intermediate and working solutions were prepared and stored at $-20^{\circ} \mathrm{C}$ for two months.

McIlvaine buffer was prepared with $10.8 \mathrm{~g}$ of citric acid, $10.93 \mathrm{~g}$ of disodium hydrogen phosphate, and $33.62 \mathrm{~g}$ of ethylenediaminetetraacetic acid disodium salt dihydrate $\left(\mathrm{C}_{10} \mathrm{H}_{14} \mathrm{~N}_{2} \mathrm{Na}_{2} \mathrm{O}_{8}\right)$. Each reagent was diluted first individually in approximately $100 \mathrm{~mL}$ of water. The EDTA solution was heated to completely dissolve the compound, avoiding reaching $50^{\circ} \mathrm{C}$. Once the three reagents were completely dissolved they were mixed and the volume was made up to $1 \mathrm{~L}$ and the $\mathrm{pH}$ adjusted to $\mathrm{pH} 4$.

2.2. Analysis by HPLC-MS/MS. HPLC-MS/MS determination of tetracyclines was performed according to a previously reported method [18]. The HPLC-MS/MS consisted of an HPLC Alliance 2795 and a MS Quattro Premier XE triple quadrupole (Waters, Manchester, UK) controlled by the software Masslynx 4.1 (Waters, Manchester, UK). The chromatographic analyses were performed by injecting $25 \mu \mathrm{L}$ of extract into a Sunfire C18 column $(150 \times 2.1 \mathrm{~mm}$ i.d., $5.0 \mathrm{~mm})$ (Waters, Manchester, UK). Mobile phases A and B were mixed on a gradient mode and with a flow rate of $0.25 \mathrm{~mL} / \mathrm{min}$. Autosampler and column temperature was set at 8 and $35^{\circ} \mathrm{C}$.

An electrospray ionisation (ESI) probe was set up on the triple quadrupole MS to evaporate the mobile phase coming from the HPLC and to ionise the tetracyclines. Analytes were detected in positive-ion mode and under the following conditions: capillary voltage $3 \mathrm{kV}$, source temperature $120^{\circ} \mathrm{C}$, desolvation temperature $350^{\circ} \mathrm{C}$, cone gas flow $49 \mathrm{~L} \mathrm{~h}^{-1}$, and 
desolvation gas flow $650 \mathrm{~L} \mathrm{~h}^{-1}$. As indicated in the Decision $2002 / 657 / E C$, tetracyclines were identified on the basis of their selected reaction monitoring (SRM) transition and their retention time $(\mathrm{Rt})$.

2.3. Sample Extraction. The sample extraction procedure was based on our previous work [17]. Firstly, before sample analysis, matrix matched calibration curves were prepared. Different volumes of the tetracycline working standard solution were added to $2 \mathrm{~g}$ feed samples (exempt of tetracycline) and shaken in the dark for $30 \mathrm{~min}$. Concentrations of tetracycline in the matrix matched feed samples were 0, 400, 800, 1200, 1600 , and $1600 \mu \mathrm{g} / \mathrm{kg}$.

To extract the tetracyclines from the feed samples, $2 \mathrm{~g}$ of grounded feed, $8 \mathrm{~mL}$ of McIlvaine buffer, $300 \mu \mathrm{L}$ of TCA, and $0,1 \mathrm{~mL}$ of IS working solution were added to a $50 \mathrm{~mL}$ polypropylene tube. After shaking the sample in the dark for $10 \mathrm{~min}, 6 \mathrm{~mL}$ of ethyl acetate was added and the samples were shaken in an orbital shaker at $200 \mathrm{rpm}$ for $20 \mathrm{~min}$. After 15 of centrifugation at $4500 \mathrm{rpm}$, in a model 5415D centrifuge (Eppendorf, Hamburg, Germany), $2 \mathrm{~mL}$ of the supernatant was transferred to a $10 \mathrm{~mL}$ amber conical tube and evaporated to dryness, in a turboevaporator model Turbo Vap II de Zyrmark (Hopkinton, MA, USA). The final residue was dissolved in $0.5 \mathrm{~mL}$ of a mixture of mobile phase components (90A:10B) and vortexed. To filtrate the final extract Ultrafree-MC centrifugal filter (Millipore, MA, US) was employed and centrifuged at $9000 \mathrm{rpm}$ for $10 \mathrm{~min}$. The filtrate was transferred into an HPLC vial which contained a $0.3 \mathrm{~mL}$ microinsert and stored at $-20^{\circ} \mathrm{C}$; analyses were conducted within 3 days.

2.4. Validation according to the Decision 2002/657/EC. The guidelines used to validate the method and to interpret the results were those established in the Commission Decision 2002/657/EC. The decision establishes criteria and procedures for the validation of analytical methods to ensure the quality and comparability of analytical results generated by official laboratories.

Aspects such us trueness/recovery, precision (under repeatability and reproducibility conditions), specificity, and applicability/ruggedness/stability of the method were investigated. Trueness and the other validation parameters were assessed through recovery of additions of known amounts of tetracyclines in blank feed samples (except tetracyclines) as no certified reference material exists for this type of analysis and following the recommendation included in the Decision 2002/657/EC.

For validation one batch of matrix-matched samples was prepared with 21 samples fortified with tetracyclines at six concentrations $(0,400,800,1200,1600$, and $4000 \mu \mathrm{g} / \mathrm{kg})$. For concentrations 400,800 , and $1200 \mu \mathrm{g} / \mathrm{kg}$ six replicated samples were prepared with only one sample for the remaining concentrations $(0,1600$, and $4000 \mu \mathrm{g} / \mathrm{kg})$. Samples spiked with tetracyclines were shaken $10 \mathrm{~min}$ for homogenization. The whole procedure with 21 samples was repeated twice on two different days. Additionally, two reagent samples were prepared for control, a blank reagent (containing only the reagents) and fortified reagent (containing $1200 \mu \mathrm{g} / \mathrm{kg}$ of tetracycline and the reagents).
To conduct the validation three batches of matrixmatched samples were prepared; each batch consisted of 21 samples fortified with tetracyclines at $0,400,800,1200,1600$, and $4000 \mu \mathrm{g} / \mathrm{kg}$. While for levels of 400,800 , and $1200 \mu \mathrm{g} / \mathrm{kg}$ six replicated samples were prepared, only one sample was used for levels of 0,1600 , and $4000 \mu \mathrm{g} / \mathrm{kg}$. After fortification, and prior to extraction, samples were shaken on an orbital shaker at $200 \mathrm{rpm}$ for $10 \mathrm{~min}$. After the samples extraction procedure explained above was applied. As well as the 21 matrix-matched samples, with each batch of samples two additional "samples" were prepared with reagents (no feed); one was spiked with tetracycline at $1200 \mu \mathrm{g} / \mathrm{kg}$ (fortified reagents) and the other was not spiked with tetracyclines (blank reagent).

Additionally, 20 feed samples were analysed to determine selectivity/specificity. Ten were spiked with tetracyclines at a validation level $(800 \mu \mathrm{g} / \mathrm{kg})$ and with $400 \mu \mathrm{g} / \mathrm{kg}$ of three antimicrobial drugs commonly used in food animal production (sulfadiazine, sulfamethoxazol, and trimethoprim). The other ten samples were analysed without adding any veterinary drugs (Decision 2002/657/EC).

The decision limit (CC $\alpha)$ and detection capability $(\mathrm{CC} \beta)$ were determined as described by Freitas et al., following the Decision 2002/657/EC requirements and applying the validation level of $800 \mu \mathrm{g} / \mathrm{kg}$.

2.5. Sample Collection and Analysis. Nonmedicated feed samples were collected from 50 milk farms located in Galicia, Spain, to investigate the presence of tetracycline residues in feed that are being consumed by cows that are producing milk daily. Additionally, samples were supplied by feed manufacturers to investigate carry-over levels of tetracyclines after making medicated feed. The sampling procedures in both cases were conducted following the requirement of the Regulation 691/2013 [19]. Feed samples were stored at room temperature and in the dark with the objective of using similar store conditions compared to that in the industry and farms.

The main raw materials of the feed samples according to the labels were corn genetically modified (between 36 and $15 \%)$, soy flour produced from soya been genetically modified (present in some samples, between 4 and 38\%), colza flour (present in some samples, between 3 and 47\%), and barley (present in some samples, between 6 and 18\%); each feed sample had a particular composition which normally depends on the manufacture.

On the other proximate composition the feed samples were crude protein (between 18 and 26\%), crude fibre (between 4 and 10\%), oil and fat crude (between 2.5 and 6\%), crude ash (between 6 and 10\%), and sodium (between 0.7 and $4 \%)$.

\section{Results and Discussion}

3.1. Method Optimisation. Tetracyclines have more than three hydroxyl groups that are easily ionisable by ESI to enhance their detection. Therefore, ESI is commonly used for the ionisation of tetracyclines when analysis is conducted by HPLC-MS/MS, independently of the matrix type [14, 20-24]. To optimise the MS parameters for a high MS signal response, 
TABLE 1: Retention time (Rt), cone voltage (CV), collision energy, precursor, and product ions employed for ion identification.

\begin{tabular}{|c|c|c|c|c|}
\hline Tetracycline & $\operatorname{Rt}(\min )$ & $\begin{array}{l}\text { Precursor }> \\
\text { production }\end{array}$ & $\mathrm{CV}$ & $\begin{array}{c}\text { Collision } \\
\text { energy }\end{array}$ \\
\hline Tetracycline & \multirow{2}{*}{12.63} & $445>410$ & 30 & 29 \\
\hline Tetracycline & & $445>154$ & 30 & 27 \\
\hline Doxycycline & \multirow{2}{*}{13.84} & $445>428$ & 30 & 20 \\
\hline Doxycycline & & $445>125$ & 30 & 27 \\
\hline Chlortetracycline & \multirow{2}{*}{11.84} & $479>462$ & 30 & 23 \\
\hline Chlortetracycline & & $479>444$ & 30 & 23 \\
\hline Oxytetracycline & \multirow{2}{*}{12.61} & $461>443$ & 30 & 20 \\
\hline Oxytetracycline & & $461>426$ & 30 & 20 \\
\hline Demeclocycline & 12.00 & $465>448$ & 30 & 17 \\
\hline
\end{tabular}

standard solutions of $1 \mu \mathrm{g} / \mathrm{mL}$ of individual tetracyclines were infused directly into the MS. During this procedure, one precursor ion and two product ions were selected for each tetracycline to conduct SRM analysis (Table 1). With the HPLC-MS/MS operating on SRM mode, two transitions and the retention time were used to achieve four identification points for each of the analytes, as required in the Decision 2002/657/EC. It should be highlighted that with other detection methods, such as diode array or a fluorescence detector, only one identification point is achieved [14] and more steps are required. The use of precursor and product ions identified in this research for tetracyclines was also reported by Boscher et al. 2010 for the analysis of these analytes in feed, royal jelly, and muscle $[14,20]$. The separation gradient could be run at ambient temperature; however, it was observed that an increase in mobile pressure can cause the system to collapse; therefore a temperature of $35^{\circ} \mathrm{C}$ is recommended.

3.2. Optimisation of the Extraction Protocol. Based on previously reported methods for tetracycline analysis in food and feed matrices [14, 25-27] different extraction protocols were tested. Firstly, simple extractions with ethyl acetate, hexane, acetonitrile, methanol, and dichloromethane were tested. However, recoveries were low, due to the tendency of tetracyclines to form chelation complexes with different cations. Therefore, initial extraction with McIlvaine/EDTA buffer was employed and gave satisfactory results, as in previously reported methods [7, 21, 28, 29]. Tetracyclines dissolved in the McIlvaine/EDTA solution were then extracted with ethyl acetate, as these two solutions are immiscible, and separation could be easily conducted as the organic phase stays on the top layer. Other authors employ SPE cartridges such as OASIS [5] for dispersive SPE [14] instead of ethyl acetate in order to purify the extract. The use of SPE was avoided to reduce time and cost of the analysis as satisfactory results were achieved with the presented method.

Animal feed is derived from a multitude of raw materials from plant and animal origin, as well as pharmaceutical and industrial sources. As feed ingredients vary depending upon the animal, that is, poultry, swine, and cattle [30], analysis of tetracyclines in different feed types could be more complicated, particularly as the fat content will vary.
The method used in this paper has been tested in feed for cattle, laying birds and chickens, rabbits, and dairy cows, and satisfactory results were obtained in all cases. These matrices were tested by preparing matrix-matched calibration curves with each type of feed, depending on the animal that was going to consume the feed.

3.3. Method Validation. Calibration curves to quantify concentrations of tetracyclines were obtained by spiking feed samples with the analytes at different concentrations. If linear regression coefficients $\left(R^{2}\right)$ were below 0.98 the extraction procedure was repeated.

Even if a signal to noise ratio $(\mathrm{S} / \mathrm{N})$ higher than 10 was achieved at $300 \mu \mathrm{g} / \mathrm{kg}$, validation was conducted at $800 \mu \mathrm{g} / \mathrm{kg}$ to provide acceptable results at $0.5,1$, and 1.5 times the validation level $(800 \mu \mathrm{g} / \mathrm{kg})$ recommended by the Decision 2002/657/EC. Figure 1 shows chromatograms of a blank sample, Figure 2 shows a blank sample spiked with all the tetracyclines at $400 \mu \mathrm{g} / \mathrm{kg}$, and Figure 3 shows the SRM transition employed for each tetracycline in one of the samples spiked at $400 \mu \mathrm{g} / \mathrm{kg}$.

Reference materials were not available; therefore trueness of the method was calculated in terms of recoveries. Results obtained during the validation are summarised in Table 2 . Recovery was low compared with other reported methods. However, the advantage of the presented extraction protocol is that it does not require solid phase extraction and the four main tetracyclines can be identified and quantified simultaneously.

Results for repeatability, calculated as the mean RDS of the $\operatorname{RSD}(n=6)$ for each concentration on each day of the validation, were below $17 \%$ for chlortetracycline, doxycycline, oxytetracycline, and tetracycline (Table 2). Results for reproducibility, calculated as the RDS of 21 samples at the same concentration, were below $23 \%$ for all tetracyclines (Table 2).

To determine selectivity/specificity, 10 blank samples and the same samples spiked with the four tetracyclines at $800 \mu \mathrm{g} / \mathrm{kg}$ were analysed. The successful quantification of tetracyclines and the absence of interfering peaks at the retention times of each analyte demonstrated the selectivity/specificity of the method.

The limit of detection (LOD) and limit of quantification (LOQ) of the method were calculated and verified with feed samples spiked with the tetracyclines at different concentrations. Based on S/N above 3 for LOD and above 10 for LOQ in matrix-matched samples, the LOD and LOQ of the method were set at 35 and $47 \mu \mathrm{g} / \mathrm{kg}$ for chlortetracycline, 40 and $60 \mu \mathrm{g} / \mathrm{kg}$ for oxytetracycline, 24 and $40 \mu \mathrm{g} / \mathrm{kg}$ for tetracycline, and 100 and $150 \mu \mathrm{g} / \mathrm{kg}$ for doxycycline.

$\mathrm{CC} \alpha$ and $\operatorname{CC} \beta$ were determined using the conditions for substances for which no permitted limit has been established. $\mathrm{CC} \alpha$ and $\mathrm{CC} \beta$ were higher than LOD and LOQ for all the compounds, meaning that tetracyclines detected at a higher level than the CC $\beta$ will be positive and levels of tetracyclines were quantifiable, without doubt. $\mathrm{CC} \alpha$ and CC $\beta$ for chlortetracycline, doxycycline, oxytetracycline, and tetracycline were below $400 \mu \mathrm{g} / \mathrm{kg}$ (Table 2).

Validation results already published have shown, in some cases, higher repeatability, reproducibility, and lower LOQ, 


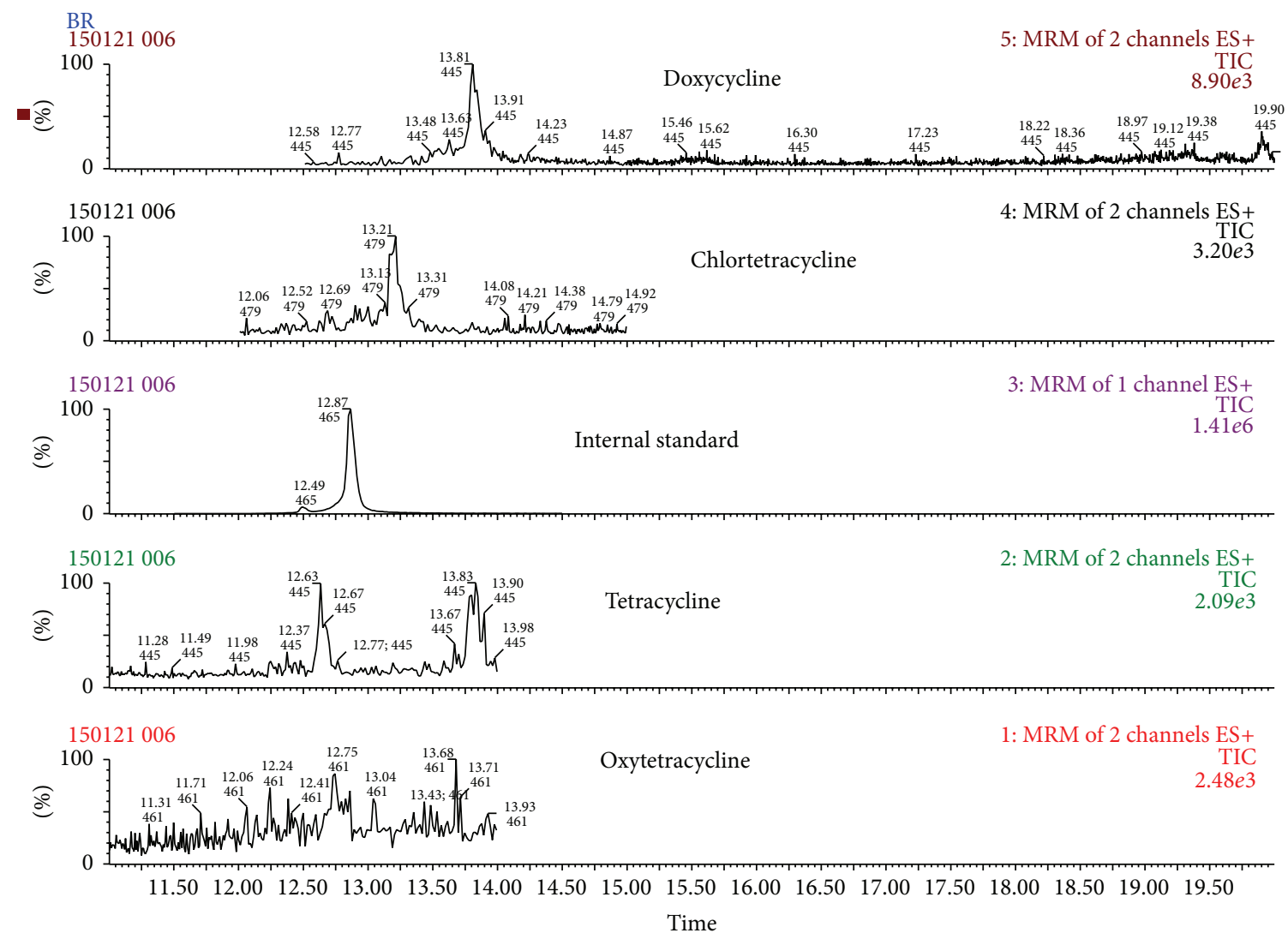

FIGURE 1: SRM chromatograms of tetracyclines in a blank sample.

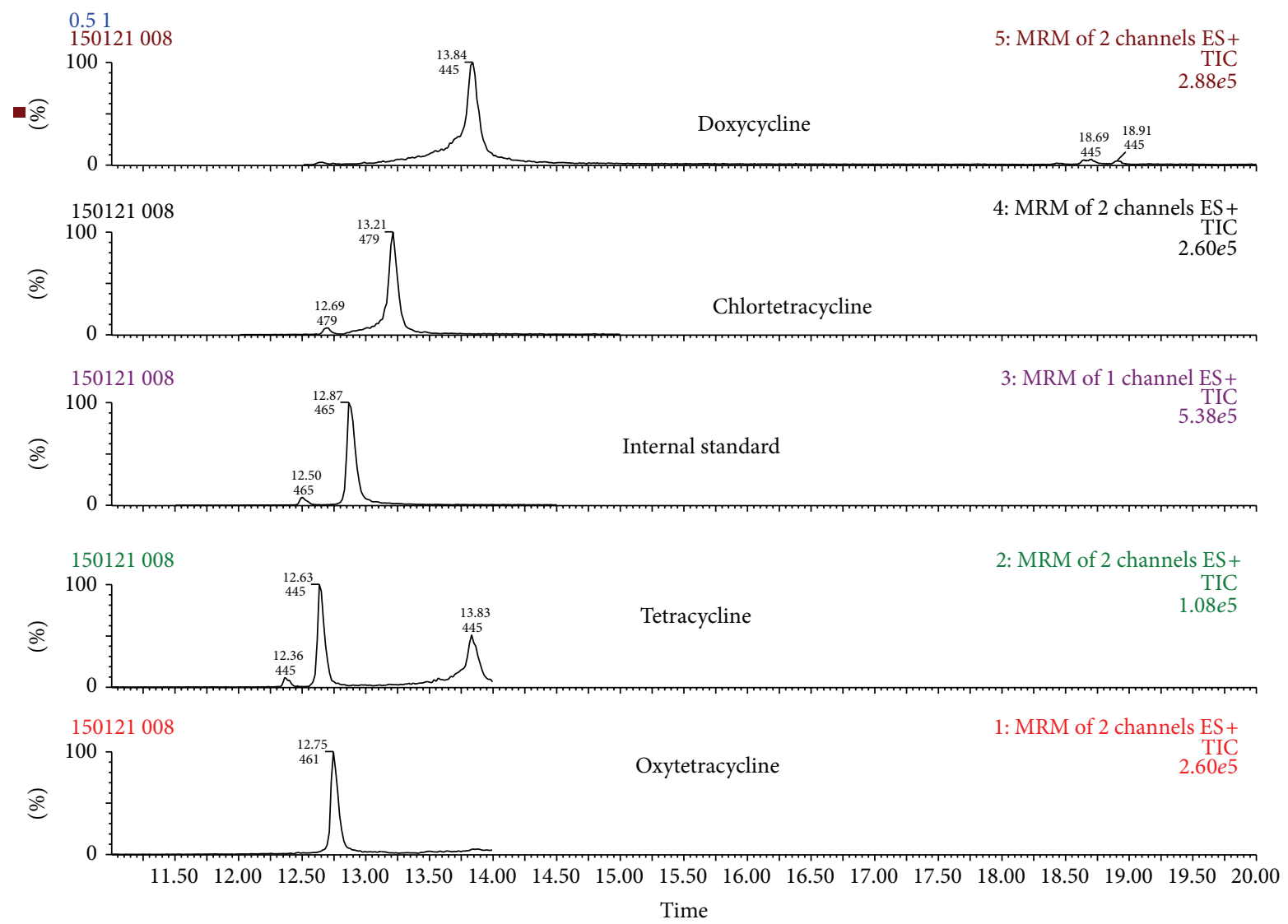

FIGURE 2: SRM chromatograms of tetracyclines in a sample fortified at $400 \mu \mathrm{g} / \mathrm{kg}$. 
TABLE 2: Recoveries (\%), repeatability (CV\%), within-laboratory reproducibility (CV\%), CC $\alpha, \mathrm{CC} \beta, \mathrm{LOD}$, and LOQ of tetracyclines.

\begin{tabular}{|c|c|c|c|c|c|c|c|c|}
\hline Tetracycline & $\mathrm{CC} \alpha(\mu \mathrm{g} / \mathrm{kg})$ & $\mathrm{CC} \beta(\mu \mathrm{g} / \mathrm{kg})$ & $\mathrm{LOD}(\mu \mathrm{g} / \mathrm{kg})$ & LOQ $(\mu \mathrm{g} / \mathrm{kg})$ & Level $(\mu \mathrm{g} / \mathrm{kg})$ & Accuracy & Repeatability & Reproducibility \\
\hline \multirow{3}{*}{ Chlortetracycline } & \multirow{3}{*}{146} & \multirow{3}{*}{249} & \multirow{3}{*}{35} & \multirow{3}{*}{47} & 400 & 89 & 11 & 13 \\
\hline & & & & & 800 & 91 & 12 & 13 \\
\hline & & & & & 1200 & 111 & 17 & 23 \\
\hline \multirow{3}{*}{ Doxycycline } & \multirow{3}{*}{205} & \multirow{3}{*}{344} & \multirow{3}{*}{100} & \multirow{3}{*}{150} & 400 & 93 & 16 & 22 \\
\hline & & & & & 800 & 113 & 15 & 17 \\
\hline & & & & & 1200 & 109 & 17 & 19 \\
\hline \multirow{3}{*}{ Oxytetracycline } & \multirow{3}{*}{198} & \multirow{3}{*}{315} & \multirow{3}{*}{40} & \multirow{3}{*}{60} & 400 & 103 & 16 & 20 \\
\hline & & & & & 800 & 90 & 12 & 22 \\
\hline & & & & & 1200 & 103 & 15 & 20 \\
\hline \multirow{3}{*}{ Tetracycline } & \multirow{3}{*}{92} & \multirow{3}{*}{164} & \multirow{3}{*}{24} & \multirow{3}{*}{40} & 400 & 78 & 12 & 13 \\
\hline & & & & & 800 & 95 & 12 & 13 \\
\hline & & & & & 1200 & 100 & 10 & 10 \\
\hline
\end{tabular}
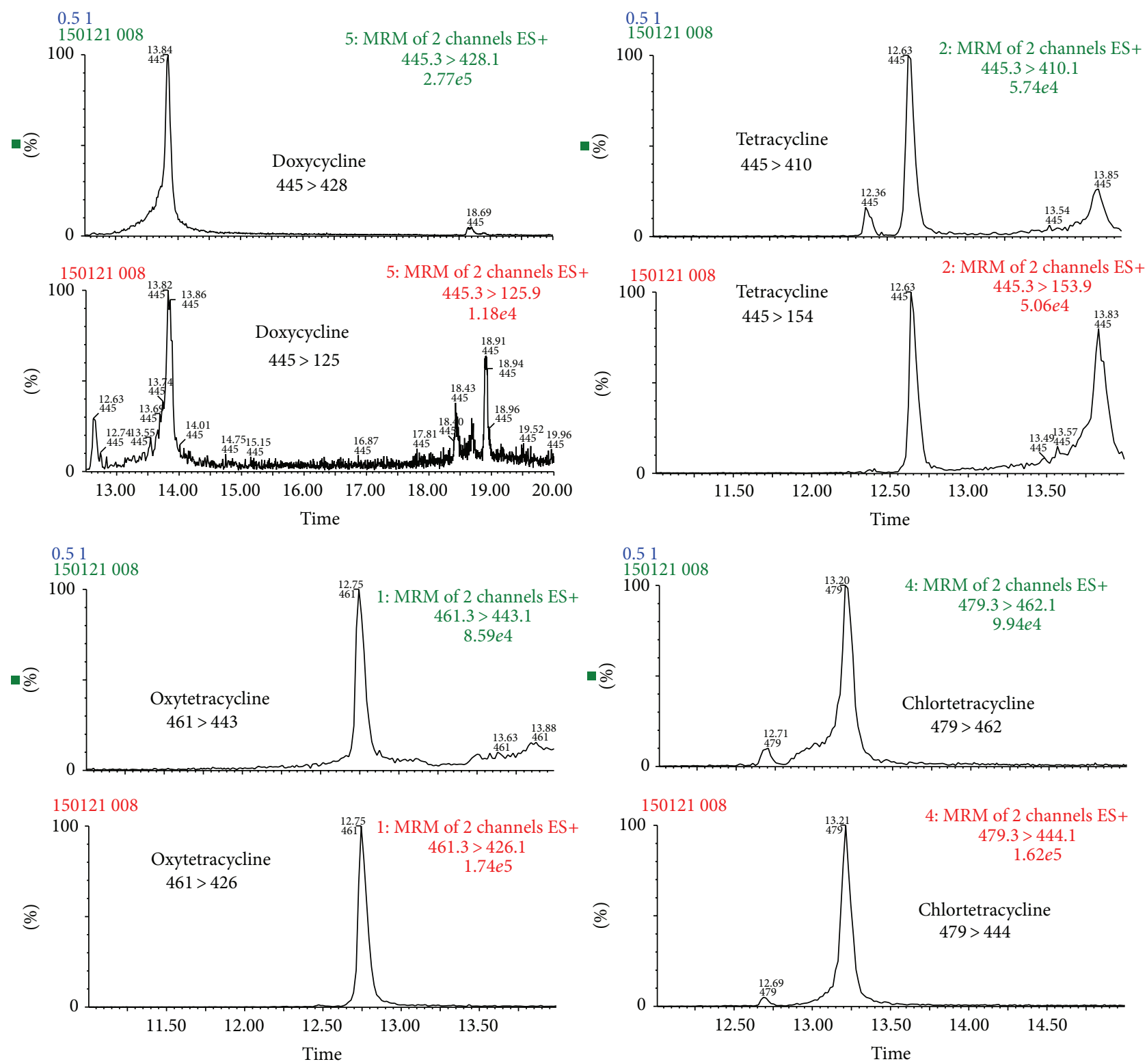

FIGURE 3: SRM chromatograms of individual tetracyclines in a sample fortified at $400 \mu \mathrm{g} / \mathrm{kg}$. 
such as the work conducted by Boscher et al. who reported RSD lower than $12 \%$ and LOQ of $20 \mu \mathrm{g} / \mathrm{kg}$. Similarly, the method reported by Stolker et al. achieved a LOQ of $0.1 \mu \mathrm{g} / \mathrm{kg}$ for doxycycline and oxytetracycline. However, it should be highlighted that none of the methods reported, based on the authors knowledge, have been validated according to the Decision 2002/657/EC.

3.4. Real Sample Collection and Analysis. From 75 feed samples investigated oxytetracycline was the tetracycline more frequently detected present in $8 \%$ of the samples $(n=6)$; its concentration range was between 90 and $400 \mathrm{mg} / \mathrm{kg}$. On the other hand, tetracycline and doxycycline were least detected. Each tetracycline was detected in individual samples and their concentrations were 150 and $110 \mathrm{mg} / \mathrm{kg}$. Chlortetracycline was the pharmaceutical detected at the highest concentration in this study, $15.14 \mathrm{mg} / \mathrm{kg}$ in feed samples for calves. It is important to highlight that the consumption of contaminated feed at level such as $15.14 \mathrm{mg} / \mathrm{kg}$ could cause food safety problems giving positive food samples.

\section{Conclusion}

Carry-over during feed manufacture has been proved for veterinary drugs such as coccidiostats and a similar case can be considered for tetracyclines, a group of antimicrobial agents commonly used in food animal production, due to its low cost.

The research work presents a simple and fast method for the analysis of the four tetracyclines regulated in the production of food of animal origin (chlortetracycline, doxycycline, oxytetracycline, and tetracycline). The method was validated according to the European guideline and successfully applied to 75 nonmedicated feed samples. Results showed the presence of tetracyclines in $15 \%$ of the samples, indicating that cross contamination occurs and maximum levels for tetracyclines may be required in the future.

\section{Competing Interests}

The authors declare that there is no conflict of interests regarding the publication of this paper.

\section{Acknowledgments}

The authors wish to thank the Consellería de Cultura, Educación e Ordenacion Universitaria, Xunta de Galicia (Project EM 2012/153), for financial support.

\section{References}

[1] Eurostat. Pocketbooks. Food: from farm to fork statistics, January 2015, http://ec.europa.eu/eurostat/documents/3930297/ 5966590/KS-32-11-743-EN.PDF/64ace8f7-ea9f-431c-81cd83ef3a601ea6.

[2] S. A. E. Kools, J. F. Moltmann, and T. Knacker, "Estimating the use of veterinary medicines in the European Union," Regulatory Toxicology and Pharmacology, vol. 50, no. 1, pp. 59-65, 2008.
[3] Regulation 124/2009/EC, "Commission regulation (EC) No. 124/2009," Official Journal of the European Communities L, vol. 40, pp. 7-11, 2009.

[4] Regulation 574/2011/EC, "Commission regulation (EC) No. 574/2011," Official Journal of the European Communities L, vol. 159, pp. 7-24, 2011.

[5] A. A. M. Stolker, V. Manti, T. Zuidema et al., "Carry-over of veterinary drugs from medicated to non-medicated feeds in commercial feed manufacturing plants," Food Additives of Contaminants: Part A, vol. 30, no. 6, pp. 1100-1107, 2013.

[6] D. J. Mevius, M. J. W. Sprenger, and H. C. Wegener, "EU conference 'the microbial threat," International Journal of Antimicrobial Agents, vol. 11, no. 2, pp. 101-105, 1999.

[7] M. M. Aguilera-Luiz, R. Romero-González, P. Plaza-Bolaños, J. L. Martínez Vidal, and A. Garrido Frenich, "Wide-scope analysis of veterinary drug and pesticide residues in animal feed by liquid chromatography coupled to quadrupole-time-of-flight mass spectrometry," Analytical and Bioanalytical Chemistry, vol. 405, no. 20, pp. 6543-6553, 2013.

[8] V. Sharma, B. K. Wadhwa, and H. J. Stan, "Multiresidue analysis of pesticides in animal feed concentrate," Bulletin of Environmental Contamination and Toxicology, vol. 74, no. 2, pp. 342-349, 2005.

[9] P. Viñas, N. Campillo, L. Carrasco, and M. Hernández-Córdoba, "Analysis of nitrofuran residues in animal feed using liquid chromatography and photodiode-array detection," Chromatographia, vol. 65, no. 1-2, pp. 85-89, 2007.

[10] H. Z. Senyuva, J. Gilbert, G. Türköz, D. Leeman, and C. Donnelly, "Analysis of deoxynivalenol, zearalenone, T-2, and HT2 toxins in animal feed by LC/MS/MS - a critical comparison of immunoaffinity column cleanup with no cleanup," Journal of AOAC International, vol. 95, no. 6, pp. 1701-1708, 2012.

[11] T. Varzakas, V. Demopoulos, and E. Manolopoulou, "Detection of aflatoxins, trichothecenes and zearalenone in food and animal feed by reversed phase HPLC and SPE," Acta Horticulturae, vol. 963, pp. 119-128, 2012.

[12] Commission Decision 2002/657/EC, “The European parliament and the Council of the European Union. Commission Decision of 12 August 2002 implementing Council Directive 96/23/EC concerning the performance of analytical methods and the interpretation of results," Official Journal of the European Communities, vol. 221, 2002.

[13] C. Van Poucke, K. De Keyser, A. Baltusnikiene, J. D. G. McEvoy, and C. Van Peteghem, "Liquid chromatographic-tandem mass spectrometric detection of banned antibacterial growth promoters in animal feed," Analytica Chimica Acta, vol. 483, no. 1-2, pp. 99-109, 2003.

[14] A. Boscher, C. Guignard, T. Pellet, L. Hoffmann, and T. Bohn, "Development of a multi-class method for the quantification of veterinary drug residues in feedingstuffs by liquid chromatography-tandem mass spectrometry," Journal of Chromatography A, vol. 1217, no. 41, pp. 6394-6404, 2010.

[15] Commission Regulation 37/2010/EU, “Commission Regulation $37 / 2010 / \mathrm{EU}$ of 22 December 2009 on pharmacologically active substances and their classification regarding maximum residue limits in foodstuffs of animal origin," Official Journal of the European Communities, vol. L 15, 2009.

[16] H. Oka, Y. Ito, and H. Matsumoto, "Chromatographic analysis of tetracycline antibiotics in foods," Journal of Chromatography A, vol. 882, no. 1-2, pp. 109-133, 2000.

[17] C. R. Anderson, H. S. Rupp, and W.-H. Wu, "Complexities in tetracycline analysis-chemistry, matrix extraction, cleanup, 
and liquid chromatography," Journal of Chromatography A, vol. 1075, no. 1-2, pp. 23-32, 2005.

[18] R. E. Gavilán, C. Nebot, J. M. Miranda et al., "Analysis of tetracyclines in medicated feed for food animal production by HPLC-MS/MS," Antibiotics, vol. 5, no. 1, pp. 1-10, 2016.

[19] Regulation 697/2013/EC, "Commission regulation (EC) No. 697/2013," Official Journal of the European Communities L, vol. 197, pp. 1-12, 2013.

[20] C. Blasco, A. D. Corcia, and Y. Picó, "Determination of tetracyclines in multi-specie animal tissues by pressurized liquid extraction and liquid chromatography-tandem mass spectrometry," Food Chemistry, vol. 116, no. 4, pp. 1005-1012, 2009.

[21] E. Cristofani, C. Antonini, G. Tovo, L. Fioroni, A. Piersanti, and R. Galarini, "A confirmatory method for the determination of tetracyclines in muscle using high-performance liquid chromatography with diode-array detection," Analytica Chimica Acta, vol. 637, no. 1-2, pp. 40-46, 2009.

[22] A. A. M. Stolker, P. Rutgers, E. Oosterink et al., "Comprehensive screening and quantification of veterinary drugs in milk using UPLC-ToF-MS," Analytical and Bioanalytical Chemistry, vol. 391, no. 6, pp. 2309-2322, 2008.

[23] M. Cherlet, M. Schelkens, S. Croubels, and P. De Backer, "Quantitative multi-residue analysis of tetracyclines and their 4epimers in pig tissues by high-performance liquid chromatography combined with positive-ion electrospray ionization mass spectrometry," Analytica Chimica Acta, vol. 492, no. 1-2, pp.199213, 2003.

[24] Z. Yue, Y. Qiu, X. Liu, and C. Ji, "Determination of multiresidues of tetracyclines and their metabolites in milk by high performance liquid chromatography-tandem positive-ion electrospray ionization mass spectrometry," Chinese Journal of Analytical Chemistry, vol. 34, no. 9, pp. 1255-1259, 2006.

[25] A. Freitas, J. Barbosa, and F. Ramos, "Multi-residue and multiclass method for the determination of antibiotics in bovine muscle by ultra-high-performance liquid chromatography tandem mass spectrometry," Meat Science, vol. 98, no. 1, pp. 58-64, 2014.

[26] M. T. Martins, J. Melo, F. Barreto et al., "A simple, fast and cheap non-SPE screening method for antibacterial residue analysis in milk and liver using liquid chromatography-tandem mass spectrometry," Talanta, vol. 129, pp. 374-383, 2014.

[27] R. Fernandez-González, M. S. García-Falcón, and J. SimalGándara, "Quantitative analysis for oxytetracycline in medicated premixes and feeds by second-derivative synchronous spectrofluorimetry," Analytica Chimica Acta, vol. 455, no. 1, pp. 143-148, 2002.

[28] A. L. Cinquina, F. Longo, G. Anastasi, L. Giannetti, and R. Cozzani, "Validation of a high-performance liquid chromatography method for the determination of oxytetracycline, tetracycline, chlortetracycline and doxycycline in bovine milk and muscle," Journal of Chromatography A, vol. 987, no. 1-2, pp. 227-233, 2003.

[29] J. Tong, Q. Rao, K. Zhu, Z. Jiang, and S. Ding, "Simultaneous determination of five tetracycline and macrolide antibiotics in feeds using HPCE," Journal of Separation Science, vol. 32, no. 2324, pp. 4254-4260, 2009.

[30] A. R. Sapkota, L. Y. Lefferts, S. McKenzie, and P. Walker, "What do we feed to food-production animals? A review of animal feed ingredients and their potential impacts on human health," Environmental Health Perspectives, vol. 115, no. 5, pp. 663-670, 2007. 

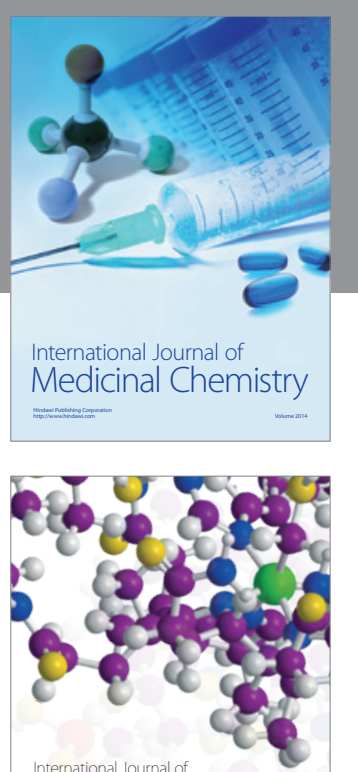

Carbohydrate Chemistry

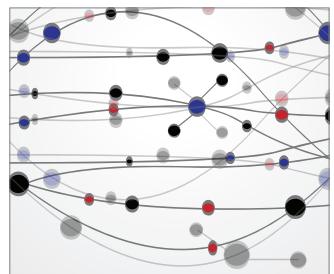

The Scientific World Journal
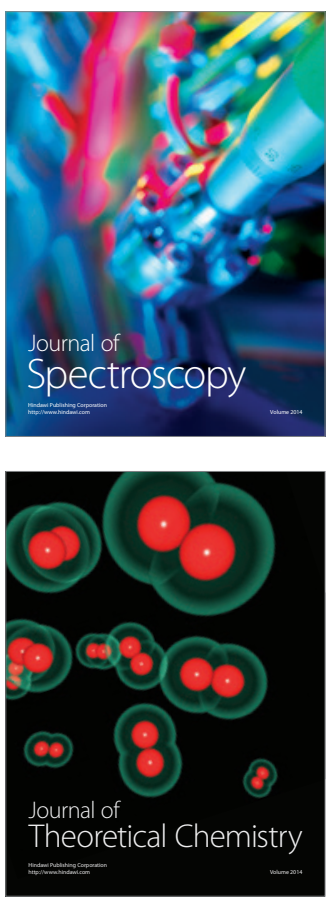
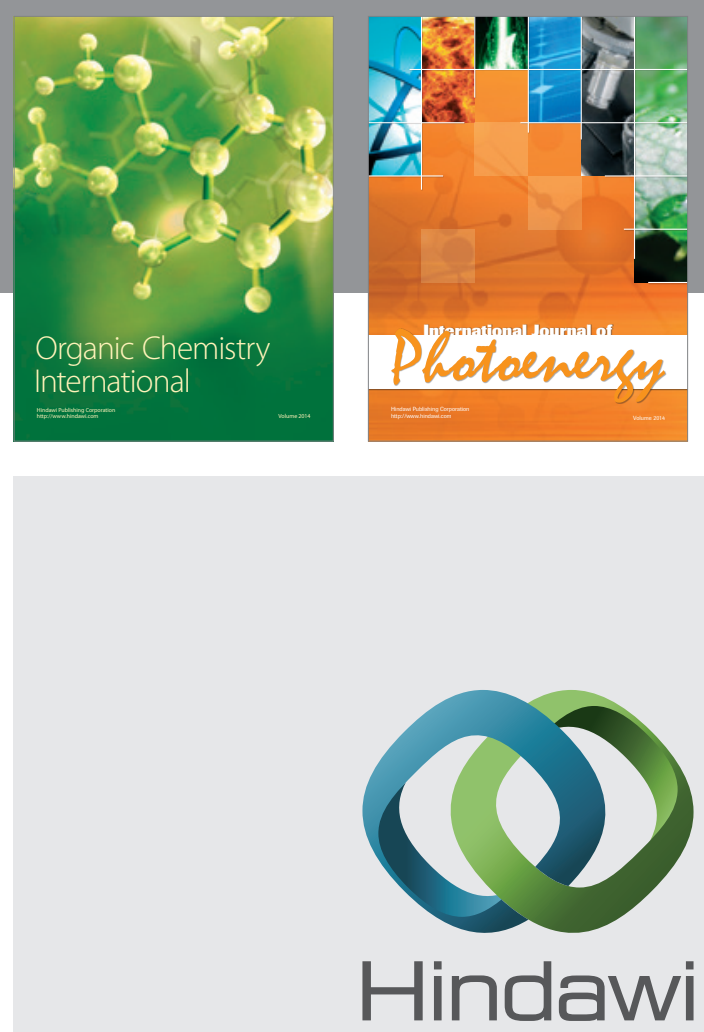

Submit your manuscripts at

http://www.hindawi.com

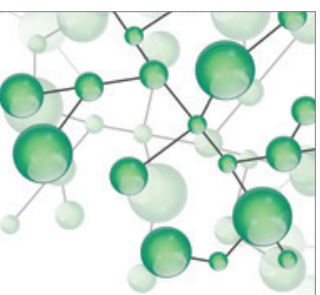

International Journal of

Inorganic Chemistry

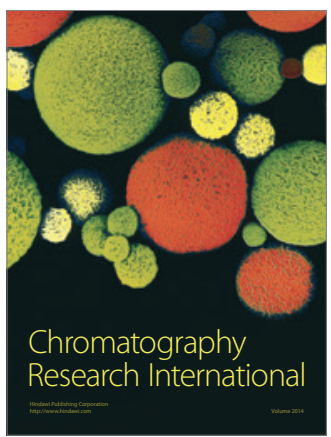

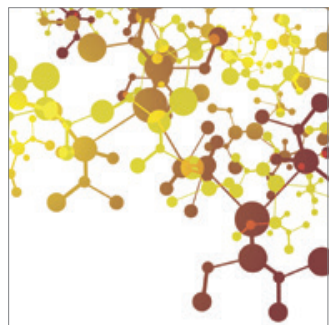

Applied Chemistry
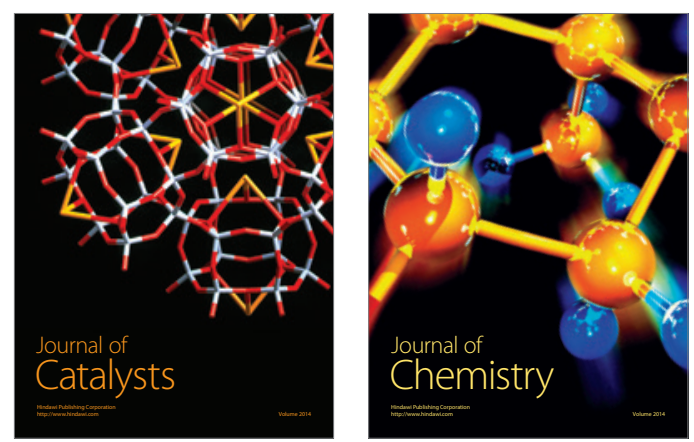
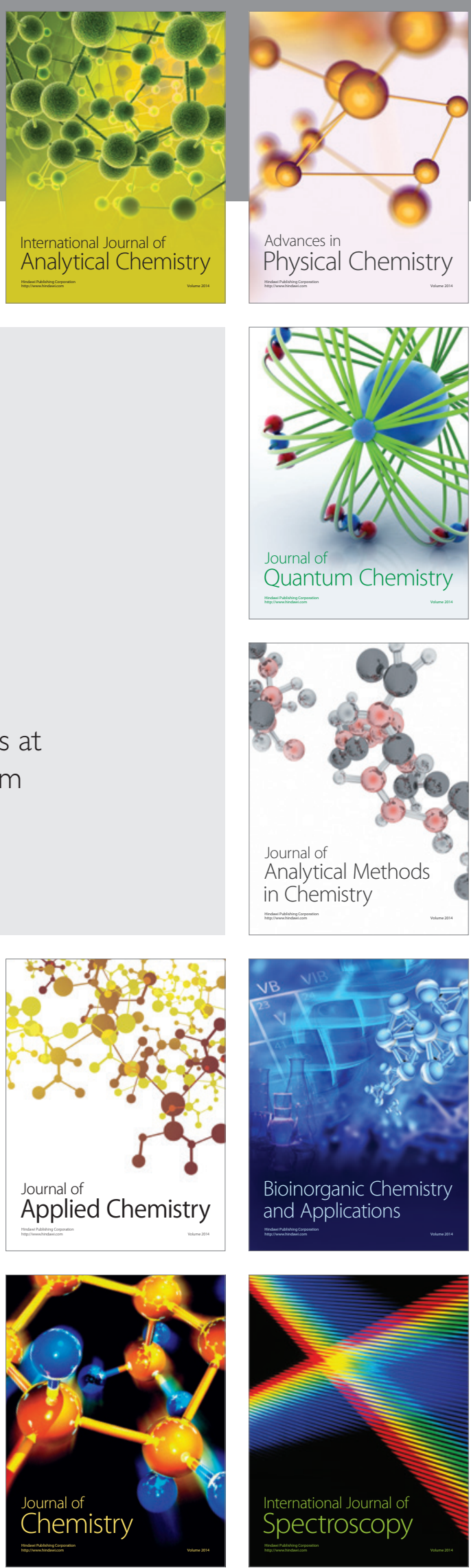